\title{
7
}

\section{Raising the Technological Level: The Scope for API, Excipients, and Biologicals Manufacture in Africa}

Joseph Fortunak, Skhumbuzo Ngozwana, Tsige Gebre-Mariam, Tiffany Ellison, Paul Watts, Martins Emeje and Frederick E. Nytko III

\section{Introduction}

This chapter discusses raising the technological scope for locally manufacturing active pharmaceutical ingredients (APIs), excipients and biologicals in Africa - a hitherto nascent industry. It also discusses African drug development and manufacturing through the standardized use of 'reverse pharmacology' to bring new treatments for neglected diseases to the point of regulatory approvals. Currently there is very modest production of APIs on the African continent, although a few significant projects exist, ${ }^{1}$ (such as LaGray in Ghana and Fine Chemicals in South Africa), or are in the planning stages. ${ }^{2}$ Generic producers in India and China supply nearly all of the APIs used in African pharmaceutical manufacturing. Most African companies cannot afford the heavy investment and research and development activities required for API production.

Two strategies are suggested to help to address these issues: first, the introduction of technology transfer (product development) packages at centres of excellence, with each package being transferred to several manufacturers; second, the use of 'leap-frogging' technologies to reduce capital investment, minimize the environmental footprint and enhance competitiveness. Leap-frogging technologies have the advantage that Africa can skip technology and investment legacy issues. Leap-frogging technologies can also narrow the gap between rich and poor economies in drug discovery/development and pharmaceutical manufacturing by 
taking advantage of the rich diversity of natural products sources and indigenous knowledge in disease treatment.

In this chapter we tackle API, excipient and biologicals manufacture by considering the science, technology and human as well as institutional and organizational capabilities needed to raise Africa's technological levels in API, excipient and FPP manufacture. This chapter is informed by the laboratory and practice experience in API, excipients and biological manufacture.

\section{APIs and excipients: important components of producing medicines}

A medicine may be broadly defined as any substance or substances used in the treatment, diagnosis, prevention, mitigation or cure of a disease. Drug molecules that exert a biological effect are known as active pharmaceutical ingredients (APIs). APIs alone are unsuitable for human use as medicines. Various additional ingredients (excipients) are formulated in combination with APIs to manufacture finished pharmaceutical products (FPPs). An FPP contains one or more APIs and is the form in which a medicine is presented to the patient. FPPs can be tablets, capsules, oral liquids (suspensions or solutions); creams/ointments for topical administration; injectable solutions; or patches, suppositories or inhaled-delivery forms. Some delivery forms are inherently easier than others to manufacture, but each combination of API and delivery form requires an individual development program to assure the identity, stability, safety, purity, potency and efficacy of the final product. The number of APIs used for the treatment of all human diseases is relatively small. The various major pharmacopoeial compendia (international, European, US or British) list fewer than 2,500 different APIs that are approved for use as medicines. APIs are formulated in order to assure their stability, uniformity and reproducibility, to enhance patient compliance and to maximize efficacy by optimizing dissolution, absorption and bioavailability.

APIs alone are unsuitable for direct patient dosing, because FPPs must be stable, convenient, palatable and uniform in their dosage and content. Since the amount of drug dissolved in stomach fluid after oral dosing is a direct measure of how much drug is available for absorption, the use of different excipients/formulations may control solubility and whether a drug is available for immediate release, extended/sustained release or even delayed release. Tablets typically contain $20-60 \%$ loading of API content by weight. Pre-formulation studies involve the evaluation and 
establishment of target ranges for the physicochemical properties of an API (salt selection, crystal form, particle size distribution [PSD] and level of hydration/solvation).

Every solid oral dosage form contains at a minimum excipients that serve as a binder, filler, lubricant and disintegrant. Excipients that serve as surfactants, compression aids, desiccants, glidants, dissolution enhancers, taste modifiers, colorants and coatings are also very often employed. Many hundreds of excipients of various types are used in pharmaceutical formulations. These ingredients are generally regarded as safe (GRAS) when present below established permissible daily exposure (PDE) limits. Quality by design (QbD) at this stage involves careful evaluation and selection of critical quality attributes (CQAs) of the API, and matching of the desired performance characteristics of the FPP with selection and grade of excipients used.

Table 7.1 shows a range of excipients commonly used in FPPs, typically used as weight percentages of a tablet, and price ranges (prices obtained from import-export data at www.infodriveindia.com). If these costs are compared with API pricing in Table 7.2, it is readily seen that excipients are normally much less expensive than APIs as a contribution to the cost of producing FPPs.

For solid oral FPP manufacturing the API and excipients are blended together, often as powders with unlike properties. Along with blending, there is a massing process of bringing these powders together to form larger particles of uniform content, known as granules. Wet granulation is possibly the most commonly used technique for granulation. Although this process is very reliable, a subsequent drying step is required that is both process intensive and must be carefully controlled so as not to cause risk of degradation. Granules are either filled into hard gelatin capsules or compressed into tablets. Tablets may be coated in order to improve their appearance, impart distinctive identifying characteristics or enhance stability. Compression into tablets does not destroy granules. The disintegration of tablets in the stomach after ingestion releases these granules at an early step in the process of dissolution and absorption of drugs. Soft gelatin capsules are filled with a solution or suspension of API(s) in a liquid-fill process, and the technology used for these formulations is somewhat specialized.

\section{Manufacturing active pharmaceutical ingredients}

Almost all small-molecule APIs are prepared by chemical synthesis, fermentation or extraction and purification of natural products from 


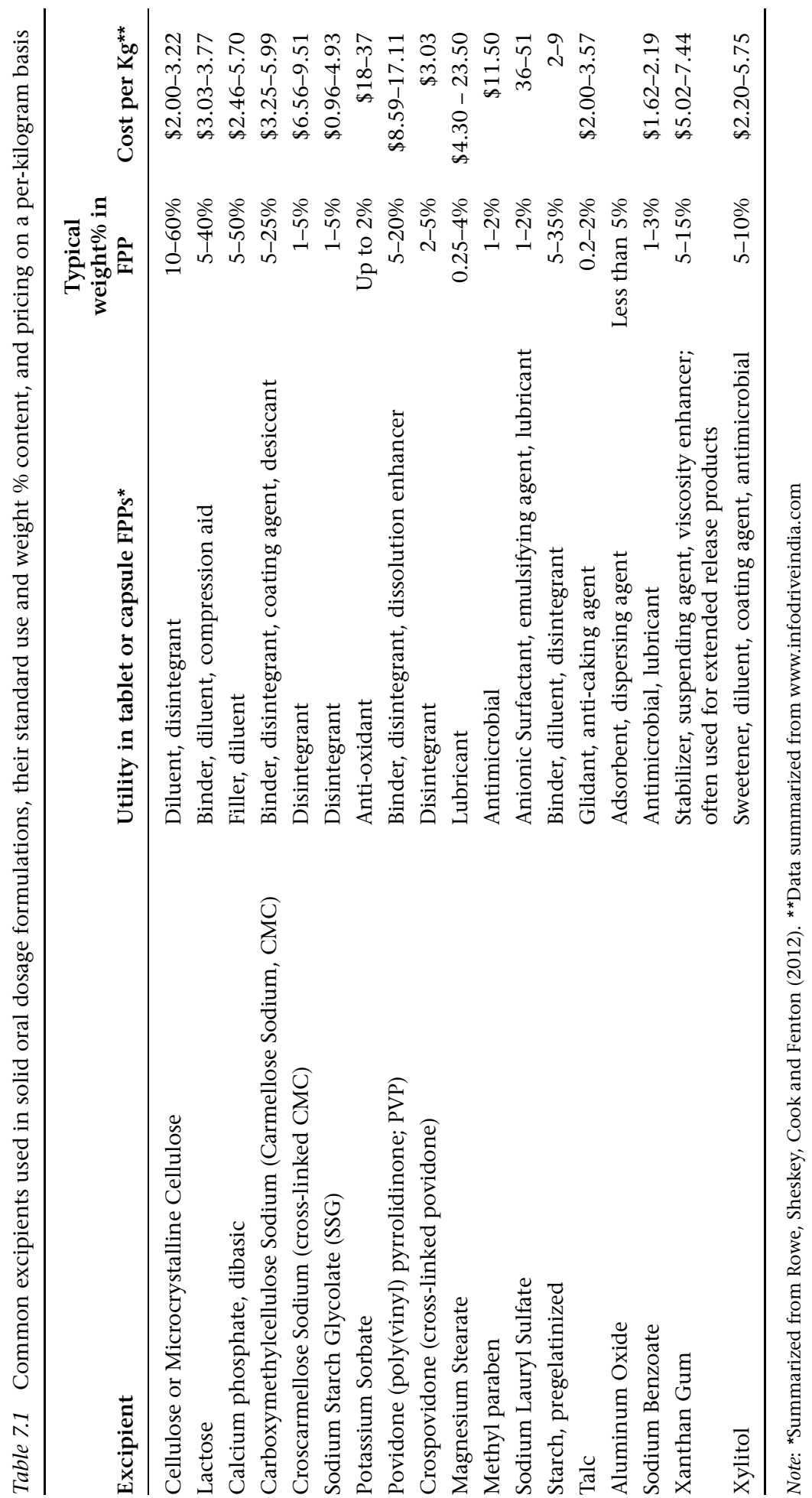


plant sources. A number of APIs are produced by some combination of these techniques. Plant cultivation and extraction of the natural product artemisinin, for example, is combined with chemical synthesis to produce the anti-malarial APIs artemether and artesunate (USNLM, 2014), as shown in Figure 7.1.

The cost of manufacturing an API depends on the raw material pricing, yield, processing time and volume efficiency of each manufacturing step. Other cost drivers are the cost of operating the manufacturing facility (overheads and labour: OHL) and capital investment required to build and equip the manufacturing plant. Overheads and labour include costs of personnel, energy, waste disposal, shipping and eventual cost of decommissioning the facility. Higher volume demand generally decreases the proportionate cost contribution of raw materials and overheads and labour. Substantial production volumes are therefore required to obtain full economy of scale (Jayaraman, 2012). Producing 1-5 metric tons per year is more expensive than producing 100 metric tons of an API in appropriately sized facilities. The efficiency of a synthesis is often quoted in terms of overall yield and as an E-factor (Sheldon, 2007) representing the kilograms of waste produced per kilogram of product manufactured. Waste management is expensive in chemical manufacturing, and increasing the overall yield or the

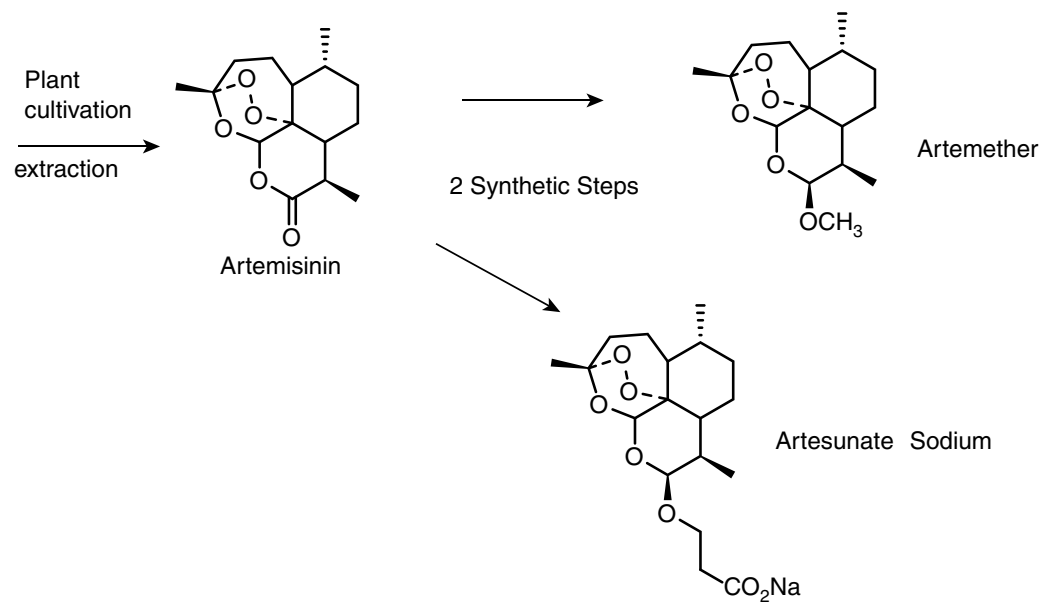

Figure 7.1 Chemical synthesis to produce the anti-malarial APIs artemether and artesunate

Source: Compiled by authors 
E-factor of an API synthesis reduces costs. With a continued growth of volume demand, improved chemistry and competition from multiple suppliers, the cost of raw materials and APIs can decrease over time. For example, the cost of the API for the HIV/AIDS drug efavirenz at launch by the originator company (Dupont Pharmaceuticals) in 1998 was about $\$ 1,600 / \mathrm{kg}$ at a first-year demand of about 150 tons (Personal recollection from the author, JF). Generic producers in India estimated their API costs at about $\$ 1,100 / \mathrm{kg}$ upon first generic introduction in 2005 (Personal communication, Mr D.R. Rao, Cipla). Currently, the API can be purchased for about $\$ 120 / \mathrm{kg}$, a 13 -fold decrease in cost of API in 17 years (import-export data at www.infodriveIndia.com). Notably, the volume demand of efavirenz in 2015 is also estimated at about 2,000 tons, since this drug is a standard component of first-line AIDS treatment in low- and middle-income countries.

As a rule of thumb, the overall cost of raw materials for an efficient process will represent about $50-70 \%$ of the total cost of API manufacturing, signifying the important role of smart procurement. This rule of thumb is applicable to high-volume generic production of synthetic APIs. Smaller-volume APIs - those produced by fermentation or natural products extraction, or APIs used in sterile products - can have much higher proportionate cost contributions for overheads and labour and capital investment. The synthetic route of manufacturing the HIV/AIDS API tenofovir disoproxil fumarate (TDF) (Figure 7.2) is a typical example. The key raw materials - adenine, (R)-propylene carbonate (RPC), diethyl p-toluenesulfonyl(oxymethyl)phosphonate (DESMP), chloromethyl isopropyl carbonate (CMIC) and fumaric acid - contribute to the molecular structure of the API. Each step uses multiple additional materials as solvents or reagents, which are omitted from this simplified synthesis scheme.

The final crystallization or precipitation step of API manufacturing is the stage at which the physicochemical properties of an API are determined and the last stage at which related substances (impurities) can be removed or reduced to acceptable limits. The crystalline form and PSD of an API are often critical to the formulation, dissolution, absorption and bioavailability of a drug. Bioavailability is the fraction of a drug dose that reaches systemic circulation (blood plasma) upon human dosing (USFDA). By definition, any drug is $100 \%$ bioavailable when administered by injection.

API cost reductions reflect improvements in production efficiency, procurement and economies of scale. The overall yield of TDF (Figure 7.2) from adenine was less than $25 \%$ when the generic product 

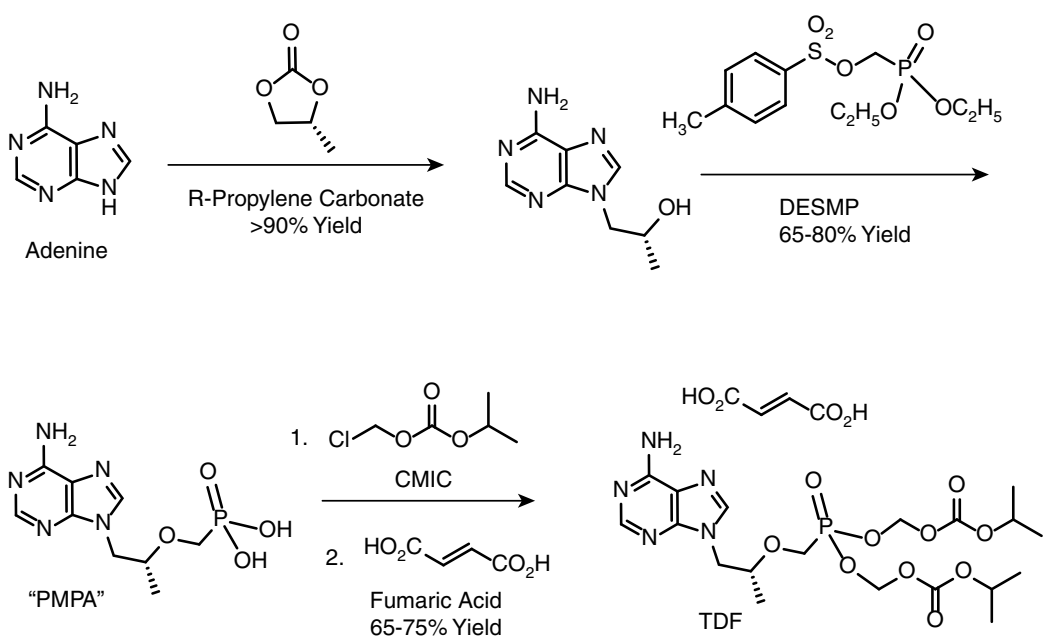

Figure 7.2 Synthetic route for the manufacture of the API tenofovir disoproxil fumarate

Source: Compiled by the author

was launched in 2006; it is currently in the range of $43-54 \%$, a doubling of efficiencies. During this time the API pricing of TDF has decreased by $65 \%$ from more than $\$ 600 / \mathrm{kg}$ to about $\$ 210 / \mathrm{kg}$. Undoubtedly a lot of research and development effort went into improving yields, reducing use of solvents and reagents and decreasing processing times (Ripin et al., 2010). Improved procurement - a critical finance capability (see Chapter 13) - greatly reduced the cost of most of the key raw materials; this was probably the biggest contributor to cost reductions (Jayaraman, 2012). Table 7.2 provides a range for the per-kilogram pricing of the key raw materials for TDF synthesis. These prices are given for purchasing ton-scale amounts of each raw material in the timeframe of JanuaryDecember 2014 (import-export data available at www.infodriveIndia. com). When generic versions of TDF were first launched in 2006, the pricing for both RPC and DESMP, for example, was more than $\$ 20 / \mathrm{kg}$.

API costs are often the largest component of the overall cost of a corresponding finished pharmaceutical product (FPP). API producers generally operate on smaller margins than FPP producers. A well-established, competitive market for anti-retroviral (ARV) APIs for the treatment of HIV/AIDS in low- and middle-income countries illustrates this. Target margins for generic APIs are typically $20-33 \%$. The actual margins on 
Table 7.2 Raw Materials that contribute to the structure of the API tenofovir disoproxil fumarate (TDF) and their current commercial pricing

\begin{tabular}{lccc}
\hline Raw Material & $\begin{array}{c}\text { Price per Kg } \\
\text { (ton scale) }\end{array}$ & $\begin{array}{c}\text { Kgs used per Kg } \\
\text { of TDF produced }\end{array}$ & $\begin{array}{c}\text { Raw Material. } \\
\text { Price Contribution } \\
\text { / kg of TDF }\end{array}$ \\
\hline Adenine & $\$ 34.34-38.97$ & 0.64 & $\$ 21.98-24.94$ \\
R-Propylene & $\$ 7.88-9.75$ & 0.50 & $\$ 3.94-4.88$ \\
Carbonate (RPC) & & 1.22 & $\$ 7.81-8.52$ \\
DESMP & $\$ 6.40-6.98$ & 1.61 & $\$ 14.15-14.88$ \\
CMIC & $\$ 8.79-9.24$ & 0.23 & $\$ 0.35-0.43$ \\
Fumaric Acid & $\$ 1.53-1.87$ & & $\$ 48.23-53.65$ \\
Total: & & & \\
\hline
\end{tabular}

Source: Compiled by the author using data from www.infodriveIndia.com, April 2012-July 2014.

ARV drugs with several approved suppliers, however, can be far lower. It is generally agreed that margins for the well-established ARV APIs zidovudine (AZT) and lamivudine (3TC) do not exceed 10\%.

\section{Can Africa expand local production of APIs?}

APIs are sold in bulk, typically as powder in a drum. Global demand for very common APIs such as paracetamol, erythromycin or ibuprofen is as high as 60,000 tons per year. Volume demand for most APIs is in the range of 10 to 500 tons per year. Economies of scale are important for both API and FPP production. A manufacturing facility must be operating at close to its full capacity to maximize operational efficiency. Companies that manufacture both APIs and FPPs possess a competitive market advantage because there is one less commercial transaction where profit is taken in the value chain.

In order to be successful, African API production must be priced similarly to that of Indian and Chinese competitors. The quality must also match or exceed the standards of competitor products in the market. Procurement information is becoming widely available, and therefore African producers potentially have access to knowledge of the best available pricing for raw materials. African producers are potentially able to obtain competitive pricing on APIs and key raw materials, because these items are not sold in huge quantities on a single-transaction basis. For solvents and reagents that require very large-volume purchases to obtain 
best pricing, however, African producers can be at a disadvantage relative to Indian and Chinese counterparts.

There are many products, however, for which it is realistic that Africa production can be price-competitive with India and China. Low-dose APIs are one set of medicines. Entecavir, for example, is a very useful product for the treatment of hepatitis B virus (HBV). Entecavir is also a very important drug for Africa, since the continent bears a very large share of the global burden for HBV. Entecavir is delivered at a dose of $0.5 \mathrm{mg} /$ day. At this extremely low dose, the API contribution to FPP pricing is potentially so small that the only important cost contribution to manufacturing an FPP is the inherent efficiency of formulating the tablet FPP. African pharmaceutical manufacturers do not, however, presently hold in their organizations the experienced, knowledgeable scientific staff to optimize API production processes in the same manner as Indian and Chinese producers are able to do.

African manufacturing of APIs and FPPs might be successful for a large range of moderately priced products that are no longer of high priority for Indian generics manufacturers. Many Indian companies target exports of more lucrative FPPs (such as gleevec or imatinib for cancer) and target high-priced markets in the US and Europe. For well-established generic products manufactured in large volumes, it is quite conceivable that African companies can purchase APIs at equal pricing to Indian FPP producers (Table 7.2). Metformin (diabetes), naproxen sodium (analgesic), amoxicillin (anti-infective), metronidazole (anthelmintic) and lumefantrine (malaria) are examples. Under these circumstances, local manufacturing may actually be less expensive than importation because of differences in added-on pricing due to shipping and import duties. This aspect of pharmaceutical pricing is commonly overlooked. The associated costs of shipping and tariffs or other duties associated with importation of APIs or FPPs can readily amount to a 30\% add-on for pricing; this can potentially create a pricing advantage for local manufacturing. International donor agencies that purchase essential medicines for Africa and African national procurement programmes often do not take into account this potential advantage for local manufacturing when issuing tender offers for purchase.

\section{'Leap-frogging' technologies as opportunities for local API manufacture}

Leap-frogging technologies are advances in manufacturing technology that provide large improvements over current techniques and avoid 
legacies of industrial organic growth. These advances can be related to chemistry, formulations or techniques for manufacturing. Global pharmaceutical companies are traditionally late adopters of new technologies because manufacturing costs are not closely tied to their product pricing or their profit model; furthermore, originator companies have huge investments in their current manufacturing facilities. For Indian companies, investment in new technologies can be unattractive unless it pays for the high cost of decommissioning existing 'legacy' manufacturing facilities. African companies do not largely suffer from such a high degree of captive 'legacy' investment in existing facilities. Continuous manufacturing of FPPs, for instance, is one area where African production can be both environmentally and cost-preferred versus global competition.

A number of patents and publications (Chava et al., 2014; Datta et al., 2014; Ripin et al., 2010) have disclosed information about TDF process optimizations that have reduced the API pricing by more than $70 \%$ since initial generic launch, as discussed earlier. New technologies for API production for TDF and EFV include biotechnology for the production of intermediates and 'greener' chemistry that reduces the E-factor of API production. In the case of $\mathrm{EFV}$, this reduction is from about 55 to about 12 (Bolu et al., 2012; Jian, 2008). Green chemistry (GC) includes the use of catalytic amounts of amino acids and their derivatives (organocatalysis) to reduce the number of steps and the cost of API processing. GC also includes continuous or 'flow' processing (Hebrault, 2014). Both of these technologies are in early stages of implementation and their full impact on API pricing is not yet being felt.

Flow chemistry is being used in one step of the commercial production of an intermediate for the synthesis of the HIV-1 protease inhibitors atazanavir and darunavir (Pinho et al., 2014). Flow chemistry can be broadly summarized as an engineering approach to improved synthesis. Organocatalysis is a more chemistry-based approach to improved synthesis. Flow chemistry for commercial manufacturing is a special expertise owned by at least one African R\&D centre, the Green Center for Chemical Manufacturing. Advanced technologies are not mutually exclusive and can often be employed together to improve API manufacturing efficiencies.

Taking advantage of these leap-frogging technology opportunities implies adoption of machinery, equipment and processes with higher efficiencies and lower environmental impact. This opportunity exists for African manufacturing of APIs. However, there is need for integration of API and FPP production to accommodate the scale needed for 
API manufacture, requiring in turn financial incentives and supportive procurement to leveraging the huge intra-African medicines market.

\section{Assuring the quality of APIs: Quality management systems and GMP}

The quality of APIs and FPPs is assured by manufacturing under good manufacturing practice (GMP), including process validation, testing against previously established specifications and the demonstration of clinical bioequivalence (FDA, 2014). The investment and operating costs of a quality management system (QMS) for GMP contribute significantly to the cost of pharmaceutical production. Producers must designate and justify to regulatory authorities a starting point or stage in the API synthesis from which GMP is applied.

Janice Berger, a well-known former USFDA field inspector, often remarked: 'You're either GMP or you're NOT'. In a very real sense, this is the true. Companies cannot choose to move back and forth from GMP to non-GMP activities in the same manufacturing facility. The requirements for a QMS and documentation for GMP do not allow switches of this type. The general feeling in the industry is that additional investment in GMP compliance enables companies to gain market share and increase sales by differentiating them from their competitors based on quality. National drug regulatory agencies (NDRAs) bear the primary responsibility of working with companies to evolve their GMP practices to meet international standards (Chapter 12). The United States Pharmacopeia (USP) sponsors training programmes in GMP. Over the longer term, African nations must assume full responsibility for their own regulatory needs. The emerging Regulatory Sciences Institute (RSI) in South Africa is potentially an important player in promoting global standards for pharmaceutical manufacturing on the continent. It follows that a sustainable African generic pharmaceutical industry must be able to:

- develop products and processes meeting SRA standards of quality;

- continuously manufacture under GMP;

- compete with other quality-assured producers on price;

- operate without legal encumbrances (dependent on intellectual property).

Regulatory sciences and rigorous product development are critical for obtaining generic drug approvals by SRAs. Originator companies employ 
a very large number of skilled scientists to develop and launch new medicines. One of us (JF) managed a scientific staff of over 350 chemists and engineers to support the filing of, on average, only 1-3 new marketing applications per year. Indian and Chinese generic companies also hold very substantial staff ( 60 or more development chemists) to launch 8-12 new generic FPPs per year. Indian and Chinese generic companies also routinely outsource substantial R\&D activities to regional centres of excellence (universities or technology development centres) to leverage their in-house capabilities. It is essential to build a pool of skills with capabilities in drug registration and regulation to support research and development as well as drug authorizations.

The IPAT program at the St. Luke Foundation / Kilimanjaro School of Pharmacy in Moshi, Tanzania is a UN ANDI Centre of Excellence training pharmaceutical and NDRA experts in GMP drug manufacturing, regulatory sciences and leap-frogging technologies since 2008. It was awarded a USFDA 'Honor Award' in 2013 for Excellence and Innovation in Regulatory Sciences training. The IPAT programme is partially funded by UNIDO and its approach has also been adopted by the University of Ibadan School of Pharmacy.

\section{Opportunities for reverse pharmacology and traditional medicine for local drug development}

\section{Reverse pharmacology}

Reverse pharmacology (RP) integrates traditional knowledge into drug discovery, development and production (Patwardan, 2009; Wambebe, 2001). This approach identifies natural products or mixtures of natural products based on traditional knowledge combined with screening in biological assays. This green approach to drug discovery takes advantage of cultural and ethno-botanical knowledge of a region to shortcut the lengthy, expensive, resource-intense process used in rational drug design.

The 'hits' identified by reverse pharmacology are further developed into drug candidates by exploratory studies including safety and activity testing, combined with clinical (human) dosing. During clinical development, mixtures of compounds obtained as crude extracts are often used, but the classical activities of isolating compounds with biological activity and elucidating their structure remains a part of reverse pharmacology. The correlation of API content with biological activity, safety, the development and validation of analytical methods, process validation 
and the setting of specifications for the active ingredients present are all steps in the RP process.

\section{Drugs from traditional knowledge}

Phytomedicines, or herbal medicines, are plants, herbs, extracts and purified natural products used for the prevention or treatment of disease, most often identified and derived from traditional knowledge. The regulatory requirements for approving phytomedicines are lower than for new chemical entities (NCEs), although the label claims for efficacy are also much more cautious. More than $80 \%$ of people in developing countries depend on herbal medicines (Iyamu, 2003; Patwardan, 2009; Perampaladas, 2010; Wambebe, 2001; Willcox, 2011). The World Health Organization has issued guidance on (1) quality assessment (WHO, 2007), (2) pharmacovigilance (WHO, 2004) and (3) good manufacturing practices (WHO, 2007) for herbal medicines. However, processes to regulate the registration, approval and standardization of such products are only now being made universal (WHO, 2007).

Phytomedicines are typically purified by short, simple processes such as aqueous or aqueous alcoholic decoction, followed by solvent removal. This is very different from pharmaceutical natural product isolation from plants, such as the vinca alkaloids, taxol or camptothecins. These are typically present as much less than $1 \%$ of the dried weight of their respective plant sources and are purified by expensive, resource-intensive procedures.

\section{Nigerian experience with Niprisan ${ }^{\mathrm{TM}}$}

A major centre for herbal medicines exists at the National Institute for Pharmaceutical R\&D (NIPRD) in Nigeria. NIPRD utilizes a systematic approach to gathering, classifying, extracting, testing and identifying the therapeutic elements present in plant sources. A quality management system is in place at NIPRD, and standard operating procedures (SOPs) are followed by a multi-disciplinary team of scientists. This standardized approach has resulted in the launch of several effective medicines for treating malaria, sickle-cell anaemia, pain, inflammation and immunological diseases (Emeje, 2005; Emeje, 2011; Wambebe, 2001a; 2001b). The development of the herbal medicine Niprisan ${ }^{\mathrm{TM}}$ for treating sicklecell anaemia is described below.

Niprisan $^{\mathrm{TM}}$ is a combined extract of four plants traditionally used in Nigeria to prevent sickle-cell disease (SCD) (Iyamu, 2003; Wambebe, 2001). SCD is caused by a point mutation in the gene encoding for the beta globin chain of hemoglobin, distorting red blood cells into an 
elongated, 'sickled' shape. SCD causes impaired circulation, tissue and organ damage, severe anaemia and increased mortality. In vitro studies showed that Niprisan ${ }^{\mathrm{TM}}$ possesses potent anti-sickling effects. One or more of the active ingredients contained in Niprisan ${ }^{\mathrm{TM}}$ enhances the solubility of deoxy-HbS, retarding polymerization and increasing oxygen affinity (Wambebe, 2001). Controlled clinical trials conducted in Nigeria showed that Niprisan ${ }^{\mathrm{TM}}$ significantly reduces vaso-occlusion in SCD patients (Iyamu, 2003; Wambebe, 2001b).

Niprisan ${ }^{\mathrm{TM}}$ was standardized by extracting various seeds, fruits and stems from Pterocarpus osun, Sorghum bicolor, Piper guineensis and Eugenia caryophyllum. Data from animal testing and using blood from sicklecell patients showed that the standard extracts prevent cell sickling. The safety of these extracts was demonstrated by animal testing before human use to demonstrate the absence of overt toxicities and lack of tissue and organ effects (Perampaladas, 2010; Wambebe, 2001a; 2001b). An initial human safety study was carried out in twenty patients, with the product already being used by thousands of people in Nigeria in an unstandardized product presentation (Wambebe 2001a).

Clinical phase II/III trials (in sickle-cell patients) using a capsule dosage form began about 18 months after the start of the discovery program at NIPRD. In that time, the product was identified, extracts were standardized, assays were developed, safety assessment was carried out, an initial manufacturing process was identified and clinical supplies were prepared. The extraction process for Niprisan ${ }^{\mathrm{TM}}$ is quite efficient, and extraction is not destructive of the cultivated plants. The dried extract represents about $20 \%$ of the collected biomass. One kilogram of Niprisan ${ }^{\mathrm{TM}}$ is therefore obtained utilizing about 5 kilograms of biomass and 10 litres each of water and ethanol.

A randomized, double-blind, placebo-controlled, crossover trial was conducted to support the approval of Niprisan ${ }^{\mathrm{TM}}$ (Perampaladas, 2010; Wambebe, 2001b). Patients with homozygous SCD in one group took the test drug ( $12 \mathrm{mg} / \mathrm{kg}$ body weight) daily for six months before crossing over to placebo for another six months. Another group took placebo for six months before crossing over to active drug for six months. There was a one-month washout period between crossovers. Niprisan ${ }^{\mathrm{TM}}$ use reduced the mean number of crises by $55 \%$ from placebo - a highly significant improvement $(\rho<0.05)$. Patients taking Niprisan ${ }^{\mathrm{TM}}$ generally rated their health as better and reported less sickness and absenteeism versus placebo. Apart from headache (12\%), patients reported no significant adverse effects (Perampaladas, 2010; Wambebe, 2001a; 2001b). It was concluded that Niprisan ${ }^{\mathrm{TM}}$ was efficacious for the prophylactic management of SCD. 
SCD patients can, to a substantial degree, recognize the symptoms of impending SCD attacks. The occasional prophylactic use of Niprisan ${ }^{\mathrm{TM}}$ with chronic, intermittent dosing by approximately 90,000 people in Nigeria has resulted in the reduction of SCD attacks by $98 \%$ in this population.

Herbal extracts almost always contain several compounds, so the attribution of biological activity to a single particular compound can be difficult. Experience at NIPRD has shown that some phytomedicines are more active as crude extracts than as purified entities. As in the case of Niprisan ${ }^{\mathrm{TM}}$, some extracts contain multiple closely related molecules with similar biological activity. Niprisan ${ }^{\mathrm{TM}}$ was approved by the Nigerian National Agency for Food and Drug Administration and Control (NAFDAC) in 2006 (see http://nafdac.org.ng/). The estimated total discovery and development costs of Niprisan ${ }^{\mathrm{TM}}$ were approximately US\$28 million (out-of-pocket), including US\$8 million to build a manufacturing facility (Pandey, 2009). This figure is much lower than the figures for rational drug discovery and development.

The following conclusions can be offered when comparing reverse pharmacology with rational drug discovery and manufacturing:

- Medicines from traditional knowledge/reverse pharmacology can be standardized in production and demonstrated to be safe by current standards.

- It is possible to prove that phytomedicines are effective in clinical trials.

- Phytomedicines production can be much less expensive than common means of pharmaceutical production.

- Reverse pharmacology can be highly effective and much less expensive than rational drug design for discovery and development.

Not all emerging economies, especially those in Sub-Saharan Africa, can afford to extensively adopt rational drug design to discovering and developing new medicines for their unique health needs, whereas global pharmaceutical originator companies cannot invest heavily in neglected tropical diseases (NTDs). Reverse pharmacology is a much less expensive approach to drug discovery and pharmaceutical manufacturing. Failure rates in RP development are reduced by evidence of human safety from traditional use. Clinical trials using an RP approach are not as rigorous as those for rational drug discovery. Yet it is possible to design and successfully execute placebo-controlled, crossover studies to demonstrate clinical efficacy for herbal medicines. Ultimately, drugs derived from RP are 
more accessible and affordable than those from pharmaceutical originator companies, and some compromises must be made to provide new medicines for these significant, unmet medical needs. This only emphasizes the need for green chemistry in drug discovery, to make all valid forms of drug discovery much more efficient.

\section{African capacity for API pharmaceutical manufacturing}

South Africa has invested heavily in research of medical significance, with a large number of research units funded by the Medical Research Council. The country has worked to build the capacity for national pharmaceutical manufacturing of APIs and FPPs, as small-molecule biological therapeutics, largely through the Department of Trade and Industry (DTI). The national government has sustained, for example, an investment in BIOVAC for several years in an attempt both to minimize reliance on imported vaccines and to generate foreign exchange by export of vaccine products. BIOVAC is presently manufacturing the final stages of vaccine production for local and external use under license from at least one global, originator pharmaceutical company.

The only South African-based API manufacturer, Fine Chemicals Corporation (FCC) was founded in 1962 in Cape Town. At inception, FCC produced codeine phosphate, morphine sulphate and paracetamol exclusively for the South African market. The product range was expanded in the 1980s with the development of a number of generic APIs, primarily for the US market. In the 1990s, FCC invested heavily to meet emerging international standards of GMP production. Since then, the company has been successfully inspected by various regulatory authorities including the USFDA in 1996, 1999, 2002 and 2006.

The company currently has 79 installed reactors with a capacity of 125,000 litres, with individual reactor capacities ranging from 100 litres to 6,000 litres for glass-lined and stainless steel. The company has significant expertise internally and supplements this with partnerships with local and international contract research organizations and organizations with expertise in chiral separations and catalysis. The use of APIs is typically regulated by the filing of a drug master file (DMF) that fully describes the route of synthesis, controls, CQAs, testing and specifications. FCC has in excess of 150 DMF submission in more than 16 countries around the world. Therapeutic uses for locally manufactured APIs are as immunosuppressants, anticancer, muscle relaxants, anti-psychotics, anticholinergic, bronchodilators, carbonic anhydrase 
inhibitors, analgesics, antihypertensive, antianginals and anaesthetics, among others.

Bioclones was founded in 1982 with the aim of developing monoclonal antibodies and manufacturing biotechnology-derived products for human use. The company became one of the first in the world to develop recombinant human erythropoietin, which was registered by the MCC in 1997 and marketed in South Africa in 1998.

LaGray Pharmaceuticals in Nsawam, Ghana, is unique among African pharmaceutical manufacturers. LaGray was founded in 2002 by Alexandra Graham and Paul Lartey, a wife-husband team with extensive experience in drug discovery, development and manufacturing of both APIs and FPPs in the global pharmaceutical industry. Dr. Lartey is the former director of anti-infective drug discovery at Pfizer Corp. Dr. Graham held several management positions at Abbott Labs. LaGray aims to differentiate the company from its competitors by meeting international standards of GMP. LaGray products for topical use include a number of anti-infectives. The company's early approach focussed on new products whose quality could be assured without the added investment of human bioequivalence trials. LaGray has implemented advanced processes for waste management and wastewater remediation, an area which is often ignored in African pharmaceutical manufacturing. LaGray is presently cooperating with the USP (United States Pharmacopeia) to train operators - the skilled workers who do the actual pharmaceutical manufacturing, a critical training need in West Africa. LaGray also has a small-scale manufacturing facility for APIs and is producing commercial amounts of the macrolide antibiotic azithromycin in Ghana.

\section{Leap-frogging technologies and training in new technologies for competitive advantage}

Manufacturing processes for APIs typically reflect the standard of chemistry available at the time the corresponding FPPs were first registered and approved for use. Although API processes evolve over time to give improved yields, reduce solvent use and reduce manufacturing time, the routes of synthesis generally are those as registered by originator companies. Malaria drugs are one therapeutic area for which this is true. Artemisinin combination therapies (ACTs) for malaria treatment consist of older drugs that were combined in fixed-dose combination or co-packaged FPPs for use. The Global Fund and other non-governmental organizations sourced approximately 389 million ACT treatments in 2013 (UNITAID, 2013). Artesunate: amodiaquine (ASAQ) and 
dihydroartemisinin: piperaquine (DHAP) are two standard ACTs. ASAQ is the second-most used ACT behind artemether: lumefantrine (ALU).

The Kilimanjaro School of Pharmacy has trained participants in the IPAT program to synthesize these APIs during laboratory exercises in API manufacturing. Trainees have also used the API they synthesized to manufacture DHAP and ASAP FPPs in the Kilimanjaro School of Pharmacy pilot plant. One of us (JF) has created new chemistry for the synthesis of these two APIs that is used by participants in the IPAT training. One of these exercises has been published (Fortunak et al., 2013). The new chemistry utilized for this purpose has simplified the manufacturing process for amodiaquine from five steps to two steps with no intermediate isolations; this synthesis is also readily adapted for continuous-flow chemical reactors (Fortunak, 2014). The overall yield of this synthesis is about $93 \%$ as compared with $65 \%$ for the previous route. New chemistry discovered for synthesizing piperaquine has the same number of steps as the commercial synthesis, but completely eliminates the presence of a genotoxic impurity previously present at a level of more than $2 \%$ in an intermediate stage of the synthesis. This has allowed an overall improvement in the E-factor of the synthesis from more than 45-plus kilograms of waste to approximately $8 \mathrm{~kg}$ of waste generated per kilogram of API produced. The overall yield of this process again has improved, from approximately $55 \%$ to $93 \%$ on a five-kilogram demonstration scale (Fortunak, 2014).

To address the challenge of cost-competitive production of APIs in South Africa, one of us, PW, and researchers at Nelson Mandela Metropolitan University (NMMU) are working to provide a step-change in pharmaceutical manufacturing technology that will increase the availability and affordability of APIs. This step-change will result from the continuous production of APIs in small, modular units known as flow reactors. Flow reactors operate continuously on a small scale, for extended periods of time. Production carried out on a 24/7 basis reduces the size of reactors needed for production and thereby reduces the capital investment needed for production start-ups. Although flow chemistry is engineering-intensive and the individual reactors are more expensive than traditional, chemical stirred tank reactors, this upfront investment in advanced science can create substantial efficiencies in manufacturing footprint and production costs as well as capital investment. Waste generation during flow processing can often be significantly less than for traditional 'batch' processing of APIs.

Compared to stirred batch reactor methodology, the benefits of MRT/ flow chemistry are: 
- increased reaction control and reproducibility by:

- efficient mixing

- accurate control of time, temperature and pressure

- increased catalyst lifetime and activity

- enhanced safety due to:

- rapid dissipation of heat

- low reactant volumes at any given time

- real-time analytical evaluation

- lower cost due to

- higher chemical yield

- reduced material use and waste generation

- reduced investment

This system's flexibility has the potential to reduce the time and risk associated with transferring technology from research into production. This methodology is being exploited profitably by fine chemical companies in Europe. The aim of this project is to exploit this technology to reduce cost of drugs of importance to South Africa making the country more self-reliant. In the first instance NMMU are focusing on three antiHIV drugs, but the technology could readily be applied to other drugs of relevance to African needs.

\section{Ethiopia: Research on local sources of starch as an excipient}

Excipients used in FPPs may originate from natural sources, chemical synthesis or biotechnology. Since excipients are ingested every time a patient takes an FPP, safety, quality and price are important. Most excipients are generally regarded as safe (GRAS) for humans because of a long history of use in the food, flavourings and cosmetics industries. One of us (TGM) is researching the development of inexpensive local sources of excipients in Ethiopia for pharmaceutical production, with a focus on starch and starch derivatives, gums and resins.

There is a huge potential for the development of new and alternative local sources of excipients in Africa. Starch is used as a component in the manufacture of pharmaceuticals, food, textiles, paper and adhesives. The use of native starch or its modified forms is based on its adhesive, thickening, gelling and film-forming properties. Starches are readily available, low cost and can be processed to provide high-quality materials of varying grades for a variety of uses. Although starch is a major component in most plants, the main commercial sources are the seeds 
of cereal grains (maize, wheat and rice), tubers (potato) and certain roots (sweet potato, cassava and arrow roots). Over the last ten years, native (enset) starch has been produced on commercial scale in Ethiopia for the food and beverage industries.

The worldwide market for industrial starches is expanding, and the industrial trend is towards the use of commercially viable raw materials other than maize, wheat and potato, which have competing food uses. Some tropical plants including enset, dioscorea, cassava and godare have been investigated. Enset starch extracted from boulla provided a 99\% yield (recovery) of available starch on dry weight basis. Scanning electron microscopy (SEM) of enset starch granules showed characteristic morphology that was somewhat angular and elliptical. Laser diffraction studies revealed normal granule size distribution with a mean particle size of $46 \mu \mathrm{m}$ (Gebre-Mariam and Schmidt, 1996a). Evaluated as a binder and disintegrant for compressed tablets, enset starch was found to compare favourably with potato and maize starches (Gebre-Mariam and Nikolayev, 1993; Gebre-Mariam and Schmidt, 1996b).

Super-disintegrants are modified polymers that rapidly absorb water and swell in the aqueous environment of the stomach. Sodium starch glycolate (SSG) is the sodium salt of the carboxymethyl ether of native starch. SSG rapidly absorbs water and swells, causing tablets and capsules containing these excipients to break apart rapidly, enhancing their release properties for improved drug absorption. Enset starch was modified into sodium starch glycolate and evaluated as a super-disintegrant in compressed tablets. SSG of enset starch was found to be at least as efficient as commercial grades of SGG, Primojel ${ }^{\circledR}$ or Croscarmellose sodium. In soluble tablet formulations, sodium starch glycolate of enset was more efficient than Primojel ${ }^{\circledR}$ or Ac-Di-Sol ${ }^{\circledR}$. Tablets prepared with sodium starch glycolate of enset exhibited shorter disintegration times and faster rates of swelling and water uptake. Similarly, the sodium starch glycolate of dioscorea starch was found to be more efficient than EXPT ${ }^{\circledast}$ (the 'Explo-tab' brand of SSG) in soluble and insoluble tablet formulations (Gebre-Mariam et al., 1996b).

Comparative studies of the starch in tablet formulations indicated dioscorea starch has practically identical binding and disintegrating ability to that of potato starch (Gebre-Mariam and Schmidt, 1998). Drug release studies from matrix tablets revealed that the rate of release changed from rapid release to sustained release as the degree of substitution increased. Dissolution studies showed that enset starch acetates with high degree of substitution could act as matrix-forming agents in tablets, where the release of a drug can be significantly sustained (Nigussu et al., 
2013). Efforts are under way to progress the work for adoption in local pharmaceutical production.

\section{Conclusion: building skills for API manufacture and drug discovery}

African pharmaceutical companies largely employ excellent scientists - their staff are often the equal of scientists anywhere. A major gap, however, is one of experience. The USFDA commonly notes that an appropriate combination of 'education, training, and experience' is needed to operate under GMP. Another challenge for pharmaceutical production is that African companies rarely are able to apply substantial resources to developing new products. It is common for companies to purchase technical development packages from Indian generics producers as the source of their process descriptions. These packages are not nearly as detailed as needed for the full process understanding and control needed for SRA approvals. It is also difficult for African companies to expend equivalent resources on laboratory and pilot scale equipment and on preparing pilot- and commercial-scale development batches to optimize their manufacturing processes.

This chapter pinpoints two important strategies by which existing capabilities can be leveraged to support the emergence of a sustainable API, excipients and generic pharmaceutical industry on the African continent. Both of these approaches are suitable for implementation by regional centres of excellence (CoEs) in drug development and regulatory sciences. Regional CoEs are recognized by the PMPA and by the United Nations African Initiative for New Drugs and Diagnostics (ANDI) Program as important for developing African capabilities in pharmaceutical innovation.

The first of these strategies is to develop education and training curricula at universities and academic Institutions. These programs must initially borrow from the experience of external experts to reduce the gap in hands-on experience in manufacturing technologies API, excipients and FPP as well as regulatory science. There are substantial numbers of African nationals working in important positions in global pharmaceutical companies today. Active recruitment of some of these experienced scientists to 'return home' - a process known as braingain - could be an important point of leverage for promulgating, implementing and improving international standards for product development and SRA approvals. A number of African countries have courses in pharmacy, industrial chemistry, fermentation technologies, microbiology, 
biochemistry (including immunology) and process engineering, among others. As alluded to earlier, what is missing is hands-on industrial experience and learning-by-doing (DUI) mode of innovation.

A second strategy to promote African API, excipient and FPP development is to fund regional CoEs to develop Technology Transfer Packages (TTPs) that enable pharmaceutical companies to implement quality-assured manufacturing of a specific API, excipient or FPP. If adequately funded, equipped and staffed with competent people, the CoEs could develop pharmaceutical manufacturing processes, analytical test methods and specifications for essential medicines. They can serve as clinical trial coordinators and knowledge brokers. With such an approach it should be feasible to sustainably raise the technological scope for locally manufacturing APIs, excipients and biologicals and to support a broader value chain of local pharmaceutical production on the continent.

\section{Notes}

1. See, for example, Bioclones (South Africa), manufacturing erythropoietin, http://www.bioclones.co.za/, and LaGray Chemical Company (Ghana), manufacturing azithromycin, http://www.lagraychem.com/.

2. See, for example, South Africa: Ketlaphela - South Africa Government's ARV Manufacturing Project Enters Next Phase, all Africa, 16 May 2013,http://allafrica.com/stories/201305211581.html; Sarah Wild, 'ARV plan bounces back', Mail \& Guardian, 24 May 2013, http://mg.co.za/article/2013-05-24-00-arvplan-bounces-back.

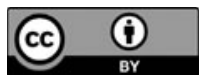

Except where otherwise noted, this work is licensed under a Creative Commons Attribution 4.0 Unported License. To view a copy of this license, visit https://creativecommons.org/version4 